\title{
Acute Kidney Injury among Salicylate Intoxication Hospitalizations in the United States
}

\author{
Charat Thongprayoon ${ }^{1}$, Tananchai Petnak ${ }^{2}$, Wisit Kaewput ${ }^{3}$, Fawad Qureshi ${ }^{1}$, Michael \\ $\mathrm{Mao}^{4}$, Aleksandra I. Pivovarova ${ }^{5}$, Boonphiphop Boonpheng ${ }^{6}$, Tarun Bathini ${ }^{7}$, Saraschandra \\ Vallabhajosyula $^{8}$, Juan Medaura ${ }^{5}$, and Wisit Cheungpasitporn ${ }^{1}$ \\ ${ }^{1}$ Mayo Clinic Division of Nephrology and Hypertension \\ ${ }^{2}$ Mahidol University Faculty of Medicine Ramathibodi Hospital Department of Medicine \\ ${ }^{3}$ Phramongkutklao College of Medicine \\ ${ }^{4}$ Mayo Clinic Hospital Jacksonville \\ ${ }^{5}$ University of Mississippi Medical Center \\ ${ }^{6}$ University of California Los Angeles Department of Medicine \\ ${ }^{7}$ University of Arizona Arizona Health Sciences Center \\ ${ }^{8}$ Emory University
}

July 31,2020

\begin{abstract}
Background: This study aimed to evaluate the risk factors and the association of acute kidney injury (AKI) with outcomes, and resource utilization in patients hospitalized due to salicylate intoxication in the United States. Methods: Hospitalized patients with a primary diagnosis of salicylate intoxication from 2003 to 2014 were identified in the National Inpatient Sample (NIS) database. End-stage kidney disease patients were excluded. The occurrence of AKI was identified using hospital diagnosis code. Clinical characteristics, in-hospital treatment, outcomes and resource utilization were compared between patients with and without AKI. Results: A total of 13,787 eligible hospital admissions were included in the analysis. AKI occurred in 1,279 $(9.3 \%)$ admissions. Older age, male sex, more recent year of hospitalization, anemia, hypertension, congestive heart failure, chronic kidney disease, volume depletion, sepsis, and ventricular arrhythmia/cardiac arrest were significantly associated with increased risk of AKI, whereas Hispanic race was associated with decreased risk. AKI was significantly associated with increased risk of organ failure, and in-hospital mortality. In addition, the need for ventilation support, blood component transfusion, renal replacement therapy, length of hospital stay, and hospitalization cost were higher in AKI patients. Conclusion: Approximately one tenth of salicylate intoxication patients developed AKI during hospitalization. AKI was associated with higher morbidity, mortality, and resource utilizations.
\end{abstract}

\section{What's already known about this topic?}

Acute kidney injury has been reported as a complication of salicylate intoxication.

\section{What does this article add?}

- Acute kidney injury occurred in about $9 \%$ of hospitalization for salicylate intoxication.

- Several clinical characteristics were identified as risk factors for acute kidney injury.

- Acute kidney injury was associated with higher morbidity, mortality, and resource utilization. 


\section{INTRODUCTION}

Salicylate-containing products are one of the most commonly used over-the-counter pharmaceuticals in the US. Acetylsalicylate and methyl salicylate are two preparations of salicylate available on the market. For many years, acetylsalicylate (aspirin) has been used as analgesic, antipyretic, and antiplatelet agents, while methyl salicylate can be found in topical ointment, herbal oil, lotions, solutions used in hot vapor and topical analgesia. ${ }^{1}$ Due to its widespread use and easy accesability, salicylates have been implicated in accidental and intentional overdose. Moreover, some salicylate containing topical preparations including herbal oils, lotions, and ointments are gaining popularity, and can potentially increase incidence of salicylate toxicity. Some of these products may contain $98-100 \%$ of methyl salicylate. Consequently, $1 \mathrm{ml}$ of solution containing approximately $5,000 \mathrm{mg}$ of methyl salicylate can potentially cause severe toxicity when ingested. ${ }^{1}$ In 2018 , approximately 27,000 toxic exposures to salicylates were reported by the American Association of Poison Control Center, with mortality rate of $0.4 \%{ }^{2}$ Among these reported cases, approximately half was due to intentional ingestion as an attempt to commit suicide.

Salicylates are absorbed in the stomach and small intestine. Most salicylates are metabolized in the liver and then excreted by the kidneys as salicyluric acid, gentisic acid, acylglucuronides, and salicylic phenolic glucuronides, while approximately 10-30\% of salicylates are excreted by kidney as free salicylic acid. Salicylate intoxication can manifest by several presentations, including hyperpnea, nausea, vomiting, dizziness, tinnitus, fever, sweating, altered mental status, coma, and organ failures. ${ }^{1}$ Acid-base abnormality is common in a setting of salicylate intoxication. Salicylates are able to directly stimulate the respiratory center resulting in hyperventilation and subsequent respiratory alkalosis. Concurrently, by interfering with aerobic metabolism salicylates cause anion-gap metabolic acidosis due to an increase of ketone bodies, lactate, and pyruvate. In addition, normal anion-gap metabolic acidosis may concomitantly occur, and is attributed to renal bicarbonate loss and chloride retention, compensating for respiratory alkalosis. ${ }^{3}$

Salicylate toxicity has been reported to affect kidneys. Several mechanisms have been proposed to be responsible for acute kidney injury (AKI). Insensible fluid loss due to increase in body temperature along with gastrointestinal losses from emesis have a potential to results in severe volume depletion, up to $6 \mathrm{~L}$ in some cases, causing renal hypoperfusion. ${ }^{4}$ In addition, salicylate-induced nephrotoxicity can manifest through acute tubular necrosis, acute interstitial nephritis, papillary necrosis, and proximal tubular dysfunction. ${ }^{5-7}$ Although AKI has been reported as a complication of salicylate intoxication, the epidemiology and impact of AKI on outcomes has been limited. Therefore, we conducted this study aiming to assess the risk factors and the association of AKI with outcomes of salicylate toxicity, and resource utilization in patients hospitalized with salicylate intoxication in the US.

\section{MATERIALS AND METHODS}

\section{Data Source}

This cohort study was conducted using the NIS database, which is the largest all-payer inpatient database in the US. The NIS database contains discharge data set from a $20 \%$ stratified sample of US hospitals with patient encounter-level information. The data set includes primary and secondary diagnosis codes as well as procedure codes in form of International Classification of Diseases, Ninth Revision (ICD-9). Sample weight is used to generate national estimates for hospitalization nationwide. The approval from institutional review board was exempted as the information was obtained from a de-identified public database.

\section{Study Population}

All hospitalized patients with the primary ICD-9 diagnosis code for salicylate intoxication (965.1) were included. Patients with ICD-9 diagnosis codes for chronic kidney disease stage 5 (585.5), and end stage renal disease (585.6) were excluded.

\section{Data Collection}

AKI was identified by the presence of ICD-9 diagnosis codes of 584.5 (acute kidney failure with lesion of 
tubular necrosis), 584.6 (acute kidney failure with lesion of renal cortical necrosis), 584.7 (acute kidney failure with lesion of renal medullary necrosis), 584.8 (acute kidney failure with other specified pathological lesion in kidney), or 584.9 (acute kidney failure, unspecified) in any of the listed diagnoses. The identification of AKI using ICD-9 diagnosis code has a specificity of $98 \%$ but a sensitivity of $17 \%$ and are likely to capture a more severe spectrum of AKI, compared with KDIGO serum creatinine-based criteria., ${ }^{8,9}$

Clinical characteristics, treatments, and outcomes during hospitalization were identified using ICD-9 codes (Table S1). Clinical characteristics included age, sex, race, year of hospitalization, alcohol consumption, certain comorbidities (obesity, anemia, diabetes mellitus, hypertension, dyslipidemia, coronary artery disease, congestive heart failure, atrial flutter/fibrillation, chronic kidney disease, and cirrhosis), and acute conditions (sepsis, volume depletion, seizure, gastrointestinal bleeding, ventricular arrhythmia/cardiac arrest). Treatments included gastric lavage, non-invasive and invasive mechanical ventilation, blood component transfusion, and renal replacement therapy. Outcomes included end-organ failure (respiratory, circulatory, liver, neurological or hematological failures), and in-hospital mortality. Resource utilization included length of hospital stay and hospitalization cost.

\section{Statistical Analysis}

Clinical characteristics, treatments, outcomes, and resource utilization between salicylate intoxication patients with and without AKI were compared using student's t-test for continuous variables, and Chi-squared test for categorical variables. Clinical characteristics associated with AKI were identified using multivariable logistic regression with backward stepwise selection. The association of AKI with clinical outcomes was evaluated using logistic regression analysis, and the association of AKI with length of hospital stay and hospitalization cost was evaluated using linear regression analysis, with pre-specified adjustment for clinical characteristics. Statistical significance was achieved when two-tailed p-value $<0.05$. SPSS statistical software (version 22.0, IBM Corporation, Armonk, NY, USA) was used for all analyses.

\section{RESULT}

Incidence of and Risk Factors for development of AKI in Patients Hospitalized with Salicylate Intoxication

Between 2003 and 2014 there were 13,805 admissions with a primary diagnosis of salicylate intoxication. A total of 18 patients with end stage renal disease were excluded, a total of 13,787 admissions were included in the study. Of these, 1,279 (9.3\%) had an AKI. Overall, among patients with salicylate intoxication, AKI was more commonly present in patients who were male, Caucasian, older and had associated comorbidities and acute conditions (Table 1 ). Multivariable analysis identified older age (OR 1.90 for age 20-29 years, 2.93 for age 30-39 years, 4.54 for age[?] 40 years; all $\mathrm{p}<0.001$ ), male sex (OR 2.59; $\mathrm{p}<0.001$ ), more recent period of hospitalization (OR 2.23 for year 2007-2010, and 3.19 for year 2011-2014; all $\mathrm{p}<0.001$ ), anemia (OR $2.31 ; \mathrm{p}<0.001$ ), hypertension (OR 1.19; $\mathrm{p}=0.03$ ), congestive heart failure (OR 1.67; $\mathrm{p}=0.002$ ), chronic kidney disease (OR 7.00; $\mathrm{p}<0.001$ ), volume depletion (OR 3.48; $\mathrm{p}<0.001$ ), sepsis (OR 5.61; $<<0.001$ ), and ventricular arrhythmia/cardiac arrest (OR $1.72 ; \mathrm{p}=0.04$ ) as clinical characteristics significantly associated with increased risk of developing AKI, and Hispanic race (OR 0.70; p-0.01) as clinical characteristics significantly associated with decreased risk of AKI (Table 2).

\section{The Association of Acute Kidney Injury with In-Hospital Treatments, and Outcomes}

Salicylate intoxication patients with AKI had higher requirment for non-invasive ventilation support (OR 3.06; $\mathrm{p}<0.001$ ), invasive mechanical ventilation (OR 3.99; $\mathrm{p}<0.001$ ), blood component transfusion (OR 2.70; $\mathrm{p}<0.001$ ), and renal replacement therapy (OR 7.25; $<<0.001$ ) than patients without AKI. After adjustment for potential confounding errors, a statistically significant association between development of an AKI with risk of organ failure, including respiratory failure (OR 4.08; $<<0.001)$, circulatory failure (OR 2.76; $\mathrm{p}<0.001$ ), liver failure (OR 6.30; $\mathrm{p}<0.001$ ), neurological failure (OR 2.05; $\mathrm{p}<0.001$ ), hematological failure (OR 3.48; $\mathrm{p}<0.001)$ and in-hospital mortality (OR 4.93; $<<0.001)($ Table 3 ).

\section{Impact of Acute Kidney Injury on Resource Utilization}


The mean length of stay in patients who developed AKI was 1.7 days longer than non-AKI patients $(\mathrm{p}<0.001)$. The mean hospitalization cost for AKI patients was significantly higher than non-AKI with additional adjusted mean hospitalization cost of $\$ 17,013(\mathrm{p}<0.001)$ (Table 3 ).

\section{DISCUSSION}

This large cohort study demonstrated risk factors associated with developing AKI in patients with salicylate intoxication and its impact on outcomes and resource utilization. Overall, 9.3\% of salicylate intoxication patients developed AKI. Factors associated with increased risk of AKI included presence of chronic kidney disease, sepsis, older age, volume depletion, more recent period of hospitalization, male sex, anemia, ventricular arrhythmia/cardiac arrest, congestive heart failure, and hypertension. Requirement of renal replacement therapy, invasive mechanical ventilation support, non-invasive ventilation support, and blood component transfusion was higher in salicylate intoxication patients with AKI. Furthermore, AKI was associated with an increased risk of organ failure and in-hospital mortality, as well as longer length of stay and higher hospitalization cost.

In our study, AKI occurred in $9.3 \%$ of patients hospitalized with salicylate toxicity, while in general the incidence of AKI range from 10 to $20 \%$ in hospitalized patients ${ }^{10-16}$ and 30 to $50 \%$ in critically ill patients. ${ }^{17,18}$ Development of AKI in salicylate intoxication is attributed to several factors, as described above. Our study identified independent factors associated with an increased risk of AKI. Pre-existing chronic kidney disease represented the highest risk factor predisposing to AKI in our study, with the adjusted odds ratio of 7.0. Not suprizingly, baseline chronic kidney disease has always been considered the risk factor for developing AKI. ${ }^{19}$ Moreover, the lower eGFR was associated with the higher risk of AKI. ${ }^{20}$ The reduction of renal reserve due to precipitating factors, such as hypotension, hypovolemia, use of nephrotoxic agents, is the probable explainion of chronic kidney disease patients being at risk for AKI. ${ }^{21}$ Sepsis also has strong association with AKI, development of AKI in critically ill patients has been attributed to sepsis in $47.5 \%$ of cases. ${ }^{22}$ Although sepsis-induced AKI is common, the pathogenesis has not been clearly explained. Several mechanisms may contribute to an increased risk of AKI in sepsis, including hemodynamic instability induced renal hypoperfusion, the injury of renal tubular endothelial cells by inflammatory mediators, microcirculation alteration, and mitochondrial dysfunction. ${ }^{23}$ Other previously reported risk factors for AKI are older age, diabetes mellitus, hypertension, congestive heart failure, use of vasopress/inotropic agents, hemodynamic instability, and anemia, requiring blood transfusion. ${ }^{24-26}$

Our study also addressed the impact of AKI among patient with salicylate intoxication on clinical outcomes. Overall, presence of AKI in salicylate intoxication patients was associated with increased in-hospital mortality. The in-hospital mortality was $4.3 \%$, with the odds ratio of $4.93(\mathrm{p}<0.001)$. AKI has also been reported to increase the mortality in several conditions, such as critical illness, acute coronary syndrome, solid organ/hematologic transplantation, cirrhosis, stroke, and cardiac surgery. ${ }^{27-35}$ In addition, greater degree of resource utilization, including renal replacement therapy, invasive/non-invasive mechanical ventilation support, and blood transfusion has been correlated with AKI. As previously described, at therapeuric doses, $10-30 \%$ of salicylate is eliminated through renal exctretion as free salicilyc acid, while the remainder is metabolized by the liver. ${ }^{1}$ In a setting of salicylate overdose, the oversaturation of hepatic metabolism, especially if accompanied by renal function impairment, results in accumulation of salicylate. This accumulation can lead to severe complications, including acidosis, acute lung injury, and central nervous system toxicity. ${ }^{5}$ Regarding pharmacokinetic physiology, salicylates are highly bound to protein at therapeutic doses. In contrast, protein binding becomes saturated at supratherapeutic dose, increasing free salicylate serum concentration. Hemodyalysis is another option for salicylate elimination. Although used only in certain settings and mainly in severe cases, hemodialysis is very efficient in eliminating salicylate and restoring acid-base balance. ${ }^{1,3}$ Some cases of salicylate intoxication require ventilation support. Acute encephalopathy, severe acidosis, and acute lung injury could be the contributing factors to respiratory failure requiring ventilation support in severe cases. ${ }^{1}$ In addition to respiratory failure, development of AKI in patients with salicylate intoxication was associated with an increased risk of other organ systems failure.

Salicylic acid has a $\mathrm{pK}_{\mathrm{a}}$ value of 3 , which essentially means that alkaline $\mathrm{pH}$ stabilizes more than $99 \%$ of 
salicylate in the ionized form. In its ionized form, the drug loses ability to easily penetrate cell membrane. On the other hand, nonionized form of salicylate in a setting of acidosis, is able to penetrate cells more readily, particularly the central nervous system, leading to neurologic toxicity. ${ }^{1,3}$ The consequence of acidosis also manifests its effects in cardiovascular system. Previous studies demonstrate that acidosis could result in myocardial dysfunction and vascular dilatation. ${ }^{36}$ Finally, salicylates also affect the liver and hematological system, resulting in liver injury, thrombocytopenia, and coagulopathy. ${ }^{37-39}$ As described above, the accumulation of saliciylate due to delayed clearance precipitated by AKI, enhances the severity of disease and leads to organ failure.

Nevertheless, our study has some limitations. According to the NIS structure, hospitalized database, we could not evaluate the long term outcome of AKI, such as renal recovery or long-term mortality. Secondly, data obtained from the NIS was extracted using diagnosis codes. Therefore, we could not conclude that these complications were consequences from AKI or concomitant complications of salicylate intoxication. The effect of co-ingestion was also not evaluated in our study, which might demonstrate some degree of effects not accounted for in this study. Finally, this study did not address the variability in outcomes between acute and chronic intoxication.

\section{CONCLUSION}

In conclusion, approximately $10 \%$ of salicylate intoxication patients developed AKI during hospitalization. Development of AKI in a setting of salicylate intoxication was associated with increased in-hospital mortality and organ failure development. Additionally, development of AKI was also found to be associated with increase in resource utilization, length of stay, and hospitalization cost. Chronic kidney disease, sepsis, older age, volume depletion, more recent year of hospitalization, male sex, anemia, ventricular arrhythmia/cardiac arrest, congestive heart failure, and hypertension were found to be associated with an increased risk of developing AKI, and therefore, could be possible predisposing factors.

\section{Disclosure}

The authors have no commercial associations that might be a conflict of interest about this article. No funding support for this article.

\section{Authors' contributions}

All authors had access to the data and a role in writing the manuscript.

\section{References}

1. Pearlman BL, Gambhir R. Salicylate intoxication: a clinical review. Postgrad Med.2009;121(4):162-168.

2. Gummin DD, Mowry JB, Spyker DA, et al. 2018 Annual Report of the American Association of Poison Control Centers' National Poison Data System (NPDS): 36th Annual Report. Clin Toxicol (Phila). 2019;57(12):1220-1413.

3. Palmer BF, Clegg DJ. Salicylate Toxicity. New England Journal of Medicine.2020;382(26):2544-2555.

4. Rupp DJ, Seaton RD, Wiegmann TB. Acute polyuric renal failure after aspirin intoxication. Arch Intern Med. 1983;143(6):1237-1238.

5. Campbell EJ, Maclaurin RE. Acute renal failure in salicylate poisoning. Br Med J.1958;1(5069):503-505.

6. Heng MC. Local necrosis and interstitial nephritis due to topical methyl salicylate and menthol. Cutis. 1987;39(5):442-444.

7. Tsimihodimos V, Psychogios N, Kakaidi V, Bairaktari E, Elisaf M. Salicylate-induced proximal tubular dysfunction. Am J Kidney Dis. 2007;50(3):463-467.

8. Grams ME, Waikar SS, MacMahon B, Whelton S, Ballew SH, Coresh J. Performance and limitations of administrative data in the identification of AKI. Clin J Am Soc Nephrol. 2014;9(4):682-689. 
9. Waikar SS, Wald R, Chertow GM, et al. Validity of International Classification of Diseases, Ninth Revision, Clinical Modification Codes for Acute Renal Failure. J Am Soc Nephrol. 2006;17(6):1688-1694.

10. Thongprayoon C, Cheungpasitporn W, Mao MA, Harrison AM, Erickson SB. Elevated admission serum calcium phosphate product as an independent risk factor for acute kidney injury in hospitalized patients. Hosp Pract (1995). 2019;47(2):73-79.

11. Thongprayoon C, Cheungpasitporn W, Mao MA, Sakhuja A, Erickson SB. Admission calcium levels and risk of acute kidney injury in hospitalised patients. Int J Clin Pract.2018;72(4):e13057.

12. Thongprayoon C, Cheungpasitporn W, Mao MA, Sakhuja A, Erickson SB. Admission hyperphosphatemia increases the risk of acute kidney injury in hospitalized patients. J Nephrol. 2018;31(2):241-247.

13. Thongprayoon C, Cheungpasitporn W, Mao MA, Sakhuja A, Kashani K. U-shape association of serum albumin level and acute kidney injury risk in hospitalized patients. PLoS One. 2018;13(6):e0199153.

14. Wang HE, Muntner P, Chertow GM, Warnock DG. Acute kidney injury and mortality in hospitalized patients. Am J Nephrol. 2012;35(4):349-355.

15. Cheungpasitporn W, Thongprayoon C, Harrison AM, Erickson SB. Admission hyperuricemia increases the risk of acute kidney injury in hospitalized patients(.). Clin Kidney J. 2016;9(1):51-56.

16. Cheungpasitporn W, Thongprayoon C, Erickson SB. Admission hypomagnesemia and hypermagnesemia increase the risk of acute kidney injury. Ren Fail. 2015;37(7):1175-1179.

17. Shao M, Li G, Sarvottam K, et al. Dyschloremia Is a Risk Factor for the Development of Acute Kidney Injury in Critically Ill Patients. PLoS One. 2016;11(8):e0160322.

18. Ronco C, Bellomo R, Kellum JA. Acute kidney injury. Lancet. 2019;394(10212):1949-1964.

19. Thongprayoon C, Hansrivijit P, Kovvuru K, et al. Diagnostics, Risk Factors, Treatment and Outcomes of Acute Kidney Injury in a New Paradigm. J Clin Med. 2020;9(4).

20. Hsu CY, Ordonez JD, Chertow GM, Fan D, McCulloch CE, Go AS. The risk of acute renal failure in patients with chronic kidney disease. Kidney Int. 2008;74(1):101-107.

21. Hsu RK, Hsu CY. The Role of Acute Kidney Injury in Chronic Kidney Disease. Semin Nephrol.2016;36(4):283-292.

22. Bagshaw SM, Uchino S, Bellomo R, et al. Septic acute kidney injury in critically ill patients: clinical characteristics and outcomes. Clin J Am Soc Nephrol.2007;2(3):431-439.

23. Peerapornratana S, Manrique-Caballero CL, Gomez H, Kellum JA. Acute kidney injury from sepsis: current concepts, epidemiology, pathophysiology, prevention and treatment. Kidney Int. 2019;96(5):10831099.

24. Cheungpasitporn W, Thongprayoon C, Kashani K. Transcatheter Aortic Valve Replacement: a Kidney's Perspective. J Renal Inj Prev. 2016;5(1):1-7.

25. Thongprayoon C, Cheungpasitporn W, Gillaspie EA, Greason KL, Kashani KB. Association of blood transfusion with acute kidney injury after transcatheter aortic valve replacement: A meta-analysis. World J Nephrol. 2016;5(5):482-488.

26. Cartin-Ceba R, Kashiouris M, Plataki M, Kor DJ, Gajic O, Casey ET. Risk factors for development of acute kidney injury in critically ill patients: a systematic review and meta-analysis of observational studies. Crit Care Res Pract.2012;2012:691013.

27. de Carvalho JR, Villela-Nogueira CA, Luiz RR, et al. Acute kidney injury network criteria as a predictor of hospital mortality in cirrhotic patients with ascites. J Clin Gastroenterol. 2012;46(3):e21-26. 
28. Kanduri SR, Cheungpasitporn W, Thongprayoon C, et al. Incidence and Mortality of Acute Kidney Injury in Patients Undergoing Hematopoietic Stem Cell Transplantation: A Systematic Review and Metaanalysis. QJM. 2020.

29. Lertjitbanjong P, Thongprayoon C, Cheungpasitporn W, et al. Acute Kidney Injury after Lung Transplantation: A Systematic Review and Meta-Analysis. J Clin Med. 2019;8(10).

30. Liborio AB, Leite TT, Neves FM, Teles F, Bezerra CT. AKI complications in critically ill patients: association with mortality rates and RRT. Clin J Am Soc Nephrol.2015;10(1):21-28.

31. Pickering JW, Blunt IRH, Than MP. Acute Kidney Injury and mortality prognosis in Acute Coronary Syndrome patients: A meta-analysis. Nephrology (Carlton).2018;23(3):237-246.

32. Thongprayoon C, Kaewput W, Thamcharoen N, et al. Incidence and Impact of Acute Kidney Injury after Liver Transplantation: A Meta-Analysis. J Clin Med. 2019;8(3).

33. Thongprayoon C, Lertjitbanjong P, Hansrivijit P, et al. Acute Kidney Injury in Patients Undergoing Cardiac Transplantation: A Meta-Analysis. Medicines (Basel). 2019;6(4).

34. Shi Q, Hong L, Mu X, Zhang C, Chen X. Meta-analysis for outcomes of acute kidney injury after cardiac surgery. Medicine (Baltimore). 2016;95(49):e5558.

35. Arnold J, Ng KP, Sims D, Gill P, Cockwell P, Ferro C. Incidence and impact on outcomes of acute kidney injury after a stroke: a systematic review and meta-analysis. BMC Nephrol. 2018;19(1):283.

36. Kimmoun A, Novy E, Auchet T, Ducrocq N, Levy B. Hemodynamic consequences of severe lactic acidosis in shock states: from bench to bedside. Crit Care. 2015;19:175.

37. Kazama I, Baba A, Endo Y, et al. Salicylate inhibits thrombopoiesis in rat megakaryocytes by changing the membrane micro-architecture. Cell Physiol Biochem.2015;35(6):2371-2382.

38. Hatten BW, Hendrickson RG. Coagulopathy and bleeding associated with salicylate toxicity. Clin Toxicol (Phila). 2020;58(1):16-19.

39. Levy G, Yaffe SJ. Clinical implications of salicylate-induced liver damage. Am J Dis Child.1975;129(12):1385-1386.

\section{Table}

Table 1 Clinical characteristics, in-hospital treatments, outcomes, and resource utilization in salicylate intoxication patients with and without acute kidney injury

Table 2 Factors associated with acute kidney injury in salicylate intoxication patients

Table 3 The association of acute kidney injury with in-hospital treatment, complications, outcomes, and resource utilization in salicylate intoxication patients

Table 1 Clinical characteristics, in-hospital treatments, outcomes, and resource utilization in salicylate intoxication patients with and without acute kidney injury

\begin{tabular}{llll}
\hline & Total & Acute Kidney Injury & No Acute Kid \\
\hline Clinical characteristics & Clinical characteristics & & \\
N (\%) & 13787 & 1279 & 12508 \\
Age (years) & $33.9 \pm 18.7$ & $48.0 \pm 19.5$ & $32.5 \pm 18.0$ \\
$<20$ & $3902(28.3)$ & $96(7.5)$ & $3806(30.5)$ \\
$20-29$ & $3227(23.4)$ & $188(14.7)$ & $3039(24.3)$ \\
$30-39$ & $1951(14.2)$ & $182(14.2)$ & $1769(14.2)$ \\
40 & $4693(34.1)$ & $812(63.5)$ & $3881(31.1)$ \\
Male & $4807(35.0)$ & $719(56.2)$ & $4088(32.8)$
\end{tabular}




\begin{tabular}{|c|c|c|c|}
\hline & Total & Acute Kidney Injury & No Acute Kidney Injury \\
\hline \multicolumn{4}{|l|}{ Race } \\
\hline Caucasian & $7721(56.0)$ & $796(62.2)$ & $6925(55.4)$ \\
\hline African American & $1386(10.1)$ & $156(12.2)$ & $1230(9.8)$ \\
\hline Hispanic & $1309(9.5)$ & $65(5.1)$ & $1244(9.9)$ \\
\hline Asian or Pacific Islander & $199(1.4)$ & $20(1.6)$ & $179(1.4)$ \\
\hline Other & $3172(23.0)$ & $242(18.9)$ & $2930(23.4)$ \\
\hline \multicolumn{4}{|l|}{ Year of hospitalization } \\
\hline $2003-2006$ & $5010(36.3)$ & $208(16.3)$ & $4802(38.4)$ \\
\hline 2007-2010 & $4425(32.1)$ & $448(35.0)$ & $3977(31.8)$ \\
\hline 2011-2014 & $4352(31.6)$ & $623(48.7)$ & $3729(29.8)$ \\
\hline Alcohol consumption & $2216(16.1)$ & $218(17.0)$ & $1998(16.0)$ \\
\hline Obesity & $520(3.8)$ & $62(4.8)$ & $458(3.7)$ \\
\hline dialysis & $885(6.4)$ & $213(16.7)$ & $672(5.4)$ \\
\hline Diabetes Mellitus & $794(5.8)$ & $155(12.1)$ & $639(5.1)$ \\
\hline Hypertension & $2123(15.4)$ & $421(32.9)$ & $1702(13.6)$ \\
\hline Dyslipidemia & $747(5.4)$ & $153(12.0)$ & $594(4.7)$ \\
\hline Coronary artery disease & $505(3.7)$ & $127(9.9)$ & $378(3.0)$ \\
\hline Congestive heart failure & $234(1.7)$ & $72(5.6)$ & $162(1.3)$ \\
\hline Atrial flutter/fibrillation & $169(1.2)$ & $46(3.6)$ & $123(1.0)$ \\
\hline Chronic kidney disease & $200(1.5)$ & $127(9.9)$ & $73(0.6)$ \\
\hline Liver cirrhosis & $116(0.8)$ & $26(2.0)$ & $90(0.7)$ \\
\hline Sepsis & $126(0.9)$ & $60(4.7)$ & $66(0.5)$ \\
\hline Volume depletion & $738(5.4)$ & $201(15.7)$ & $537(4.3)$ \\
\hline Seizure & $563(4.1)$ & $67(5.2)$ & $496(4.0)$ \\
\hline Gastrointestinal bleeding & $362(2.6)$ & $55(4.3)$ & $307(2.5)$ \\
\hline Ventricular arrhythmia/cardiac arrest & $94(0.7)$ & $26(2.0)$ & $68(0.5)$ \\
\hline Treatments & Treatments & & \\
\hline Gastric lavage & $344(2.5)$ & $25(2.0)$ & $319(2.6)$ \\
\hline Non-invasive ventilation & $64(0.5)$ & $27(2.1)$ & $37(0.3)$ \\
\hline Invasive mechanical ventilation & $759(5.5)$ & $253(19.8)$ & $506(4.0)$ \\
\hline Blood component transfusion & $354(2.6)$ & $116(9.1)$ & $238(1.9)$ \\
\hline Renal replacement therapy & $796(5.8)$ & $346(27.1)$ & $450(3.6)$ \\
\hline Complication and outcomes & Complication and outcomes & & \\
\hline Respiratory failure & $941(6.8)$ & $308(24.1)$ & $633(5.1)$ \\
\hline Circulatory failure & $480(3.5)$ & $157(12.3)$ & $323(2.6)$ \\
\hline Liver failure & $109(0.8)$ & $51(4.0)$ & $58(0.5)$ \\
\hline Neurological failure & $687(5.0)$ & $153(12.0)$ & $534(4.3)$ \\
\hline Hematological failure & $302(2.2)$ & $110(8.6)$ & $192(1.5)$ \\
\hline In-hospital mortality & $132(1.0)$ & $55(4.3)$ & $77(0.6)$ \\
\hline Resource utilization & Resource utilization & & \\
\hline Length of hospital stay (days) & $2.6 \pm 3.3$ & $5.2 \pm 6.3$ & $2.3 \pm 2.7$ \\
\hline Hospitalization cost $(\$)$ & $18089.6 \pm 29478.8$ & $42696.7 \pm 68065.2$ & $15579.8 \pm 20430.0$ \\
\hline
\end{tabular}

Continuous variables are reported as mean \pm standard deviation; categorical variables are reports as counts (percentages)

Table 2 Factors associated with acute kidney injury in salicylate intoxication patients 


\begin{tabular}{|c|c|c|c|}
\hline Variables & Univariable analysis & Univariable analysis & Multivariable analysis \\
\hline & Crude odds ratio $(95 \% \mathrm{CI})$ & $\mathrm{P}$-value & Adjusted odds ratio $(95 \% \mathrm{CI})$ \\
\hline \multicolumn{4}{|l|}{ Age (years) } \\
\hline$<20$ & 1 (reference) & & 1 (reference) \\
\hline $20-29$ & $2.45(1.91-3.15)$ & $<0.001$ & $1.90(1.47-2.45)$ \\
\hline 30-39 & $4.08(3.17-5.26)$ & $<0.001$ & $2.93(2.25-3.80)$ \\
\hline 40 & $8.30(6.68-10.30)$ & $<0.001$ & $4.54(3.59-5.74)$ \\
\hline Male & $2.63(2.34-2.96)$ & $<0.001$ & $2.59(2.28-2.95)$ \\
\hline \multicolumn{4}{|l|}{ Race } \\
\hline Caucasian & 1 (reference) & & 1 (reference) \\
\hline African American & $1.10(0.92-1.32)$ & 0.29 & $1.19(0.97-1.46)$ \\
\hline Hispanic & $0.46(0.35-0.59)$ & $<0.001$ & $0.70(0.53-0.93)$ \\
\hline Asian or Pacific Islander & $0.97(0.61-1.55)$ & 0.91 & $1.35(0.82-2.23)$ \\
\hline Other & $0.72(0.62-0.84)$ & $<0.001$ & $1.16(0.98-1.37)$ \\
\hline \multicolumn{4}{|l|}{ Year of data collection } \\
\hline 2003-2006 & 1 (reference) & & 1 (reference) \\
\hline 2007-2010 & $2.60(2.20-3.08)$ & $<0.001$ & $2.23(1.86-2.67)$ \\
\hline 2011-2014 & $3.86(3.28-4.54)$ & $<0.001$ & $3.19(2.68-3.80)$ \\
\hline Alcohol drinking & $1.08(0.93-1.26)$ & 0.32 & \\
\hline Obesity & $1.34(1.02-1.76)$ & 0.04 & \\
\hline Anemia & $3.52(2.98-4.16)$ & $<0.001$ & $2.31(1.90-2.80)$ \\
\hline Diabetes Mellitus & $2.56(2.13-3.09)$ & $<0.001$ & \\
\hline Hypertension & $3.12(2.74-3.54)$ & $<0.001$ & $1.19(1.02-1.39)$ \\
\hline Dyslipidemia & $2.73(2.26-3.29)$ & $<0.001$ & \\
\hline Coronary artery disease & $3.54(2.87-4.36)$ & $<0.001$ & \\
\hline Congestive heart failure & $4.55(3.42-6.04)$ & $<0.001$ & $1.67(1.20-2.32)$ \\
\hline Atrial flutter/fibrillation & $3.76(2.66-5.30)$ & $<0.001$ & \\
\hline Chronic kidney disease & $18.78(13.99-25.20)$ & $<0.001$ & $7.00(5.03-9.73)$ \\
\hline Liver cirrhosis & $2.86(1.84-4.45)$ & $<0.001$ & \\
\hline Volume depletion & $4.16(3.49-4.95)$ & $<0.001$ & $3.48(2.87-4.23)$ \\
\hline Sepsis & $9.28(6.51-13.23)$ & $<0.001$ & $5.61(3.79-8.29)$ \\
\hline Seizure & $1.34(1.03-1.74)$ & 0.03 & \\
\hline Gastrointestinal bleeding & $1.79(1.33-2.39)$ & $<0.001$ & \\
\hline Ventricular arrhythmia/cardiac arrest & $3.80(2.41-5.99)$ & $<0.001$ & $1.72(1.01-2.91)$ \\
\hline Gastric lavage & $0.76(0.51-1.15)$ & 0.20 & \\
\hline
\end{tabular}

Table 3 The association of acute kidney injury with in-hospital treatment, complications, outcomes, and resource utilization in salicylate intoxication patients

\begin{tabular}{|c|c|c|c|}
\hline Variables & Univariable analysis & Univariable analysis & Multivariable analysis \\
\hline & Crude odds ratio $(95 \% \mathrm{CI})$ & $\mathrm{P}$-value & Adjusted odds ratio* $(95 \% \mathrm{CI})$ \\
\hline \multicolumn{4}{|l|}{ Treatments } \\
\hline Non-invasive ventilation & $7.27(4.41-11.98)$ & $<0.001$ & $3.06(1.72-5.45)$ \\
\hline Invasive mechanical ventilation & $5.85(4.97-6.89)$ & $<0.001$ & $3.99(3.28-4.86)$ \\
\hline Blood component transfusion & $5.14(4.09-6.47)$ & $<0.001$ & $2.70(2.03-3.59)$ \\
\hline Renal replacement therapy & $9.94(8.51-11.61)$ & $<0.001$ & $7.25(6.07-8.67)$ \\
\hline \multicolumn{4}{|l|}{ Complications and outcomes } \\
\hline Respiratory failure & $5.95(5.12-6.92)$ & $<0.001$ & $4.08(3.41-4.89)$ \\
\hline Circulatory failure & $5.28(4.32-6.45)$ & $<0.001$ & $2.76(2.18-3.50)$ \\
\hline Liver failure & $8.92(6.09-13.05)$ & $<0.001$ & $6.30(4.05-9.81)$ \\
\hline
\end{tabular}




\begin{tabular}{lllll}
\hline Variables & Univariable analysis & Univariable analysis & Multivariable analysis & Mul \\
\hline Neurological failure & $3.05(2.52-3.68)$ & $<0.001$ & $2.05(1.65-2.55)$ & $<0.0$ \\
Hematological failure & $6.04(4.74-7.69)$ & $<0.001$ & $3.48(2.63-4.61)$ & $<0$. \\
In-hospital mortality & $7.24(5.10-10.28)$ & $<0.001$ & $4.93(3.15-7.71)$ & $<0$. A \\
Resource utilization & Coefficient $(95 \% \mathrm{CI})$ & $\mathrm{P}$-value & Adjusted co efficient* $(95 \% \mathrm{CI})$ & $\mathrm{P}-\mathrm{va}$ \\
Length of hospital stay (days) & $2.9(2.7-3.0)$ & $<0.001$ & $1.7(1.6-1.9)$ & $<0$. \\
Hospitalization cost $(\$)$ & $27117(25469-28765)$ & $<0.001$ & $17013(15386-18640)$ & $<0$. . \\
\hline
\end{tabular}

*adjusted for age, sex, race, year of hospitalization, alcohol drinking, obesity, anemia, diabetes mellitus, hypertension, dyslipidemia, coronary artery disease, congestive heart failure, atrial fibrillation, chronic kidney disease, liver cirrhosis, volume depletion, sepsis, seizure, gastrointestinal bleeding, ventricular arrhythmia/cardiac arrest and gastric lavage 